\title{
A brief review of recent developments in the designs that prevent bio-fouling on silicon and silicon-based materials
}

\author{
Xiaoning Zhang ${ }^{1 *}$, DaShan Brodus ${ }^{1}$, Valerie Hollimon ${ }^{1}$ and Hongmei $\mathrm{Hu}^{2}$
}

\begin{abstract}
Silicon and silicon-based materials are essential to our daily life. They are widely used in healthcare and manufacturing. However, silicon and silicon-based materials are susceptible to bio-fouling, which is of great concern in numerous applications. To date, interdisciplinary research in surface science, polymer science, biology, and engineering has led to the implementation of antifouling strategies for silicon-based materials. However, a review to discuss those antifouling strategies for silicon-based materials is lacking. In this article, we summarized two major approaches involving the functionalization of silicon and silicon-based materials with molecules exhibiting antifouling properties, and the fabrication of silicon-based materials with nano- or micro-structures. Both approaches lead to a significant reduction in bio-fouling. We critically reviewed the designs that prevent fouling due to proteins, bacteria, and marine organisms on silicon and silicon-based materials.
\end{abstract}

Keywords: Silicon, Silicon-based materials, Antifouling, Surface modification, Biomimetic

\section{Background}

Silicon and silicon-based materials are integral parts of our daily life. Silicon has widespread applications in healthcare and manufacturing due to its unique material properties, including high flexibility, low biological activity, ease of fabrication, and chemical and thermal stability [1-4]. In addition, silicone based materials, such as silicone elastomer, is also the basic constituent of tubing, microfluidic system, catheters, drains, shunts, joint implants, silastic mammary prostheses, and contact lenses [5]. Silicon-based materials, such as $\mathrm{Si}_{x} \mathrm{~N}_{4}$, have excellent fracture toughness and are chemically inert. Therefore, silicon is often used as an insulator and chemical barrier in manufacturing integrated circuits [6].

Unfortunately, silicon and silicon-based materials are susceptible to bio-fouling, which is the tendency of microbes, cells, and bacteria to physically adsorb to surfaces. This leads to deterioration of surface engineering

\footnotetext{
*Correspondence: XZhang@paine.edu

1 Department of Mathematics, Sciences and Technology, Paine College, 1235 Fifteenth Street, Augusta, GA 30901, USA

Full list of author information is available at the end of the article
}

in addition to infectious contamination [7], which are significant concerned [8]. For example, in the USA, about $40 \%$ of all bacterial infections occurring in hospitals are found to be catheter-associated urinary tract infection (CAUTI), which considerably increases the healthcare costs, the length of stay in the hospital and the antibiotic use [9-11].

Bio-fouling can also result in the growth of marine organisms on ship hulls, which is a major expense factor in naval industries. Based on the World Shipping Council's report, fuel can represent as much as $50 \%$ of a ship's total operating costs. Because the fouling causes a drag force on the ship and subsequent decreased fuel efficiency, hull fouling can increase fuel consumption, cost, and carbon dioxide emissions by as much as $40 \%$ [12]. In addition, the metabolic activity of the attached organisms can cause localized corrosion [13]. In one solution to this problem, polydimethylsiloxane (PDMS), a low energy silicone material, was used as a nontoxic alternative to conventional biocide paints.

According to previous studies, bio-fouling develops in four stages [14]. In stage 1, bio-organisms encounter a surface and form a confluent layer. In stage 2 , the 
bio-organisms multiply locally and then assemble to form a "microcolony". The third stage of this process involves the formation of macrocolonies from the embedded microcolonies. Finally, once a mature biofilm is formed, bio-organisms exit and re-enter the biofilm structure as the last stage (Fig. 1).

Significant efforts have been dedicated to modifying silicon and silicon-based materials to improve their antifouling performance. The objective was to change the properties of the materials or the immobilized molecules in ways that either limit foulant accumulation, or provide ways to remove attached foulants after adsorption saturation, such that the fouled materials can be regenerated. However, reviews of surface modification of silicon and silicon-based materials for their antifouling and antibacterial properties are lacking. Therefore, in this article, an overview of the recent work in silicon and silicon-based materials modification for antifouling purposes will be presented. We are interested in silicon-based materials such as silicone elastomer because they are appropriate to be used for medical applications.

\section{Functionalization of silicon and silicon-based substrates with antifouling molecules \\ Poly (ethylene glycol) and its derivatives}

As the most commonly-used materials for fouling resistance, poly (ethylene glycol) (PEG) and its derivatives are widely used to engineer the surface of silicon [15-17]. Their modification enables silicon to be hydrophilic, nontoxic, and biocompatible. The hydration layer surrounding the ethylene oxide chain is the reason PEG has demonstrated the ability to repel fouling materials [18]. However, the ethylene oxide chains are, over time, autooxidized in aqueous solutions, resulting in cleavage of ethylene oxide units and formation of aldehyde-terminated chains. Therefore, there are limits to its long-term application (more than 14 days). In addition, the formed aldehyde moieties may react with fouling materials, such as protein, resulting in a declination of the repellent nature of the PEG coatings [19]. Furthermore, PEG loses its protein resistance at $37{ }^{\circ} \mathrm{C}$ and above, while $37{ }^{\circ} \mathrm{C}$ is a critical temperature for many biomedical applications. It is known that repulsive ethylene oxide (EO)-protein interactions are essential to the anti-fouling efficiency of PEG. Higher temperatures $\left(37^{\circ} \mathrm{C}\right.$ and above) can cause EO monomers to alternate their configurations and affect EO-protein interactions, resulting in more protein adsorption on the surface [20].

Recently, different surface modification methods have been implemented to graft PEG molecules onto silicon and silicon-based substrates. One of these includes self-assembly, in which PEG molecules are grafted on the silicon wafers or glass slides via silanization, which is achieved by leaving silicon slides or glass slides overnight in a solution of PEG in dry toluene [21]. Research groups from Germany and Australia reported a stepwise method for the construction of PEG layers onto a silicon surface via click chemistry [22]. In their work, acetylene-terminated alkyl monolayers were first attached to non-oxidized crystalline silicon surfaces through a hydrosilylation reaction. The acetylene-terminated surfaces were then functionalized via a copper-catalyzed azide-alkyne cycloaddition reaction to generate an amine-terminated layer. Eventually, a PEG layer with high graft density was conjugated with an amine-terminated substrate via an $\mathrm{N}-\mathrm{C}$ bond (Fig. 2). The antifouling properties of this chemical-modified surface were investigated by testing adsorption of human serum albumin (HSA) and lysozyme (Lys). However, their results indicate that the surface is fully antifouling to large proteins, such as HSA, but does not completely repel the low-molecular weight protein, such as Lys. The study of protein adsorption on the surface is important, as in the clinical setting blood clots and subsequent thromboses may be more likely to develop with use of medical devices and implants.

In a report published in Langmuir [23], the copolymer, poly(TMSMA-r-PEGMA), which is comprised of an "anchor part" (trimethoxysilane) and a "function part" (PEG), was synthesized by a radical polymerization reaction. Then polymeric self-assembled monolayers (PSAMS) of poly(TMSMA-PEGMA) on $\mathrm{Si} / \mathrm{SiO}_{2}$ or glass

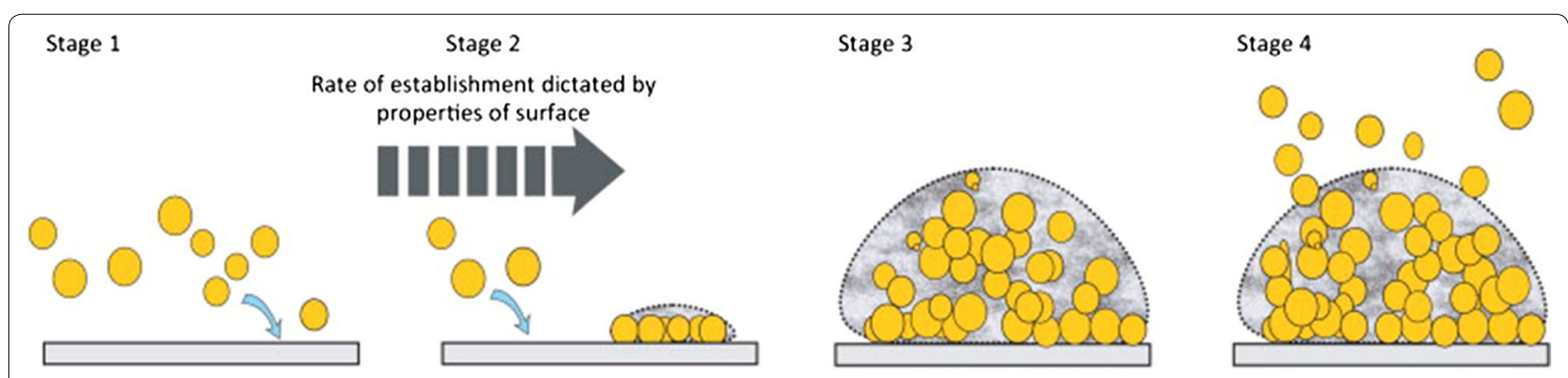

Fig. 1 The four stages of biofilm formation [16] reproduced with permission from Elsevier 


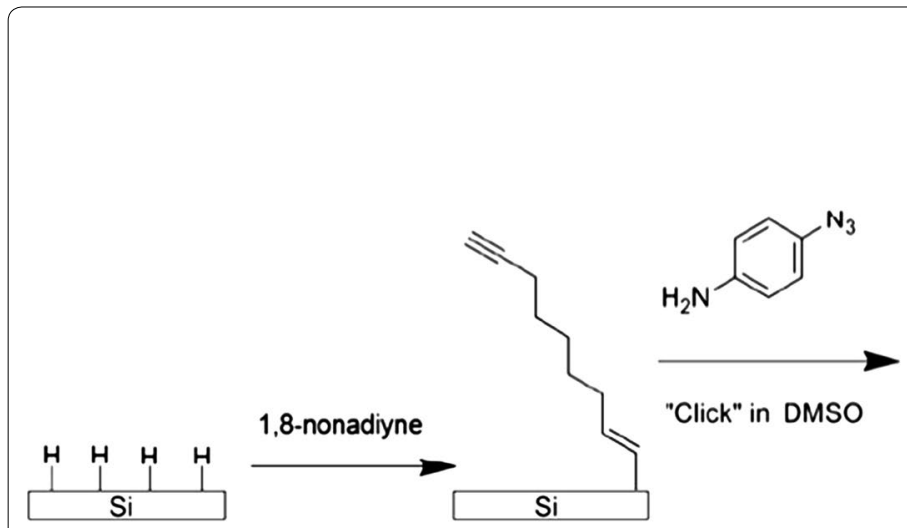

(a)

(b)

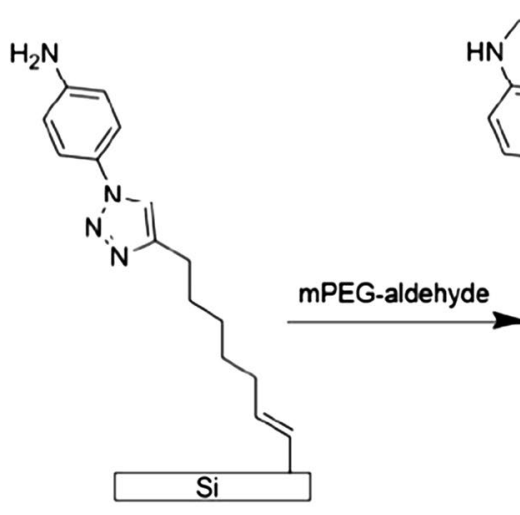

(c)

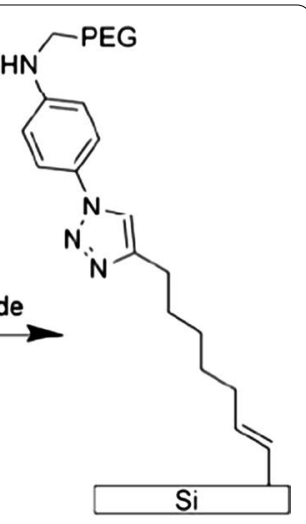

(d)

Fig. 2 Schematic diagram of grafting PEG on silicon surface via click chemistry [24] reproduced with permission from ACS Publications

substrates were prepared by immersing the substrate in a methanol solution of poly(TMSMA-PEGMA) at ambient temperature. Then the polymer-coated $\mathrm{Si} / \mathrm{SiO}_{2}$ or glass substrates were immersed in insulin, lysozyme, and fibrinogen solution to evaluate their protein resistance characteristics. The results demonstrated that the polymer-coated $\mathrm{Si} / \mathrm{SiO}_{2}$ or glass substrates have a great reduction in nonspecific protein adsorption compared to the unmodified substrates.

PEG-based materials also resist adhesion of many bacteria [24-29]. In research from Libera et al., PEG-based microgels were deposited on silicon substrates [30]. Because the average spacing between microgels on silicon substrates is approximately the same micrometer size of the bacterium itself, the bacterium experiences surface repulsiveness. In addition, the microgel modifiedsurfaces exhibit a reduced susceptibility to S. epidermidis, bacteria commonly implicated in biomaterial-associated infection. In further study, a cationic antimicrobial peptide (L5) was loaded onto the surface-bound microgels, and microgels serve as reservoirs for antimicrobial delivery. The additional loading of L5 into PEG-based microgels amplified the resistance of the microgel-modified surfaces to S. epidermidis colonization (Fig. 3). The major disadvantage associated with release coatings based on antimicrobial components is the depletion of the active species, which leads to a loss in the antimicrobial activity of the coatings with time. This problem may be addressed by using reloadable coatings. Another option is to use slow releasing coatings that release the biocide over longer periods of time [31, 32].

In another study, Voo and co-workers coated thiolfunctionalized silicone rubber, which is a commonly used catheter material, with modified PEG molecules through a Michael addition reaction. The antibacterial and antifouling properties of the polymer-modified surface

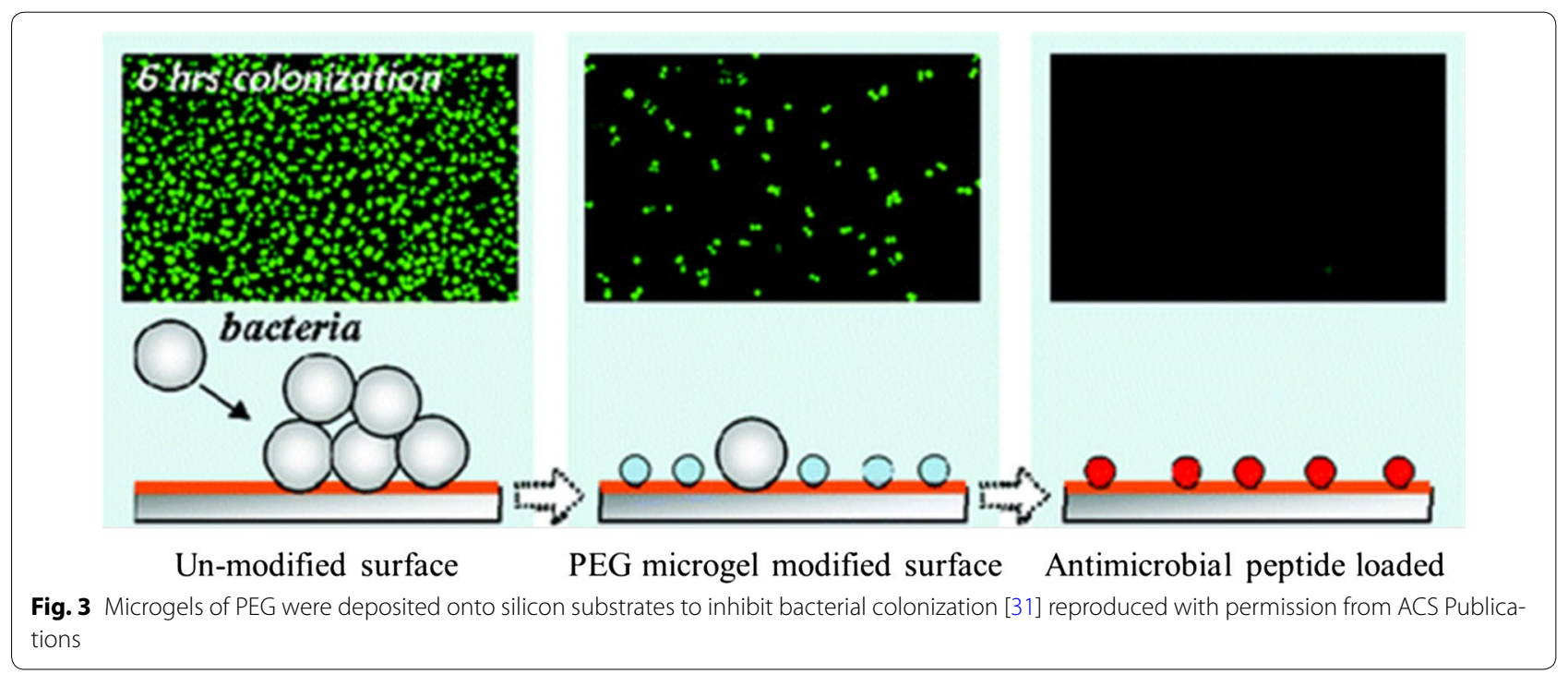


against both Gram-positive Staphylococcus aureus, which is methicillin-resistant and a major cause of the infection, and Gram-negative Escherichia coli were examined. Their results shown that the coating can prevent $S$. aureus and E. coli biofilm formation over 14 days incubation. Comparing to a high number of $S$. aureus and $E$. coli adhered onto the pristine surface after one day incubation, this coating demonstrates potential for use as antifouling coating to prevent catheter-associated infections [33].

Yang's team created PEG nonfouling anti-microbiao hydrogels, which can be applied onto catheters or other implants as a coating to prevent infections. This PEG hydrogel was fabricated via Michael addition chemistry that incorporated an antimicrobial cationic block copolymer of PEG and polycarbonate. The antimicrobial mechanism of the cationic hydrogels is proposed that the anionic cell wall/membrane of bacteria was first attracted and interacted with the cationic hydrogel surface at many fixed points via electrostatic interaction, followed by the insertion of the hydrophobic segments of hydrogel into the hydrophobic regions of the lipid membrane, inducing the leakage of the membrane and eventually resulted in cell lysis. These hydrogels were then grafted onto silicone rubber, a material used to manufacture catheters. The antimicrobial activity of hydrogel-coated rubber was investigated by exposing itself to $S$. aureus for 1 day. There were numerous viable $S$. aureus cells found on the rubber surface without coating, but no cells on the gel coated rubber surface were detected by confocal. No adverse effect of the gel was observed on the toxicity, skin sensitization and skin irritation [34].

PEG chains are also used to develop coating materials to prevent marine biofouling on a surface [35-39]. Nguyen et al. constructed new antifouling coatings by covalent grafting of methoxy-terminated PEG chains onto polysilazane polymers through a highly stable silicon carbon bond using a hydrosilylation reaction (Fig. 4) [40]. The resistance of the modified PEG materials to the adhesion of three marine bacteria species, bacillus clostridium sp. SR1, micrococcus Neisseria sp. LC1, and micrococcus Neisseria sp. SC1 was investigated. The grafting of PEG chains onto polysilazane exhibits lower levels of bacteria adhesion. Their further study demonstrated that the surface density of the PEG chains has a greater effect in inhibiting bacterial adhesion. With the increase in PEG graft density, the ability of this antifouling coating to inhibit bacterial adhesion increases. There is insufficient demonstration of long-term stability of antifouling coatings in Nguyen's article. However, the long-term fouling resistance of PEG to marine fouler was tested in Gruze and colleagues' study [41]. It was shown that PEG was oxidized in seawater and lost its antifouling properties with time and was then settled much like any other surface submersed in the spore solution.

\section{Stimuli-responsive polymers}

Stimuli-responsive, or "smart" polymers, have the extraordinary ability to change their physical and chemical state after they "detect" a change in their environments; their responses depend dramatically on their chemical composition [42]. In addition, due to the ease of modification with specific chemical functionalities, stimuli-responsive polymers have attracted additional attention for use as antifouling coatings [43-46]. Some researchers have used the properties of stimuli-responsive conformational changes to develop antifouling coating materials with self-cleaning properties [47, 48]. Compared to traditional antifouling surfaces, which are often associated with the accumulation of dead bacteria and other debris that degrade biocidal activity and provide nutrients for other colonizers [49], the stimuliresponsive polymer-modified surface is desirable for

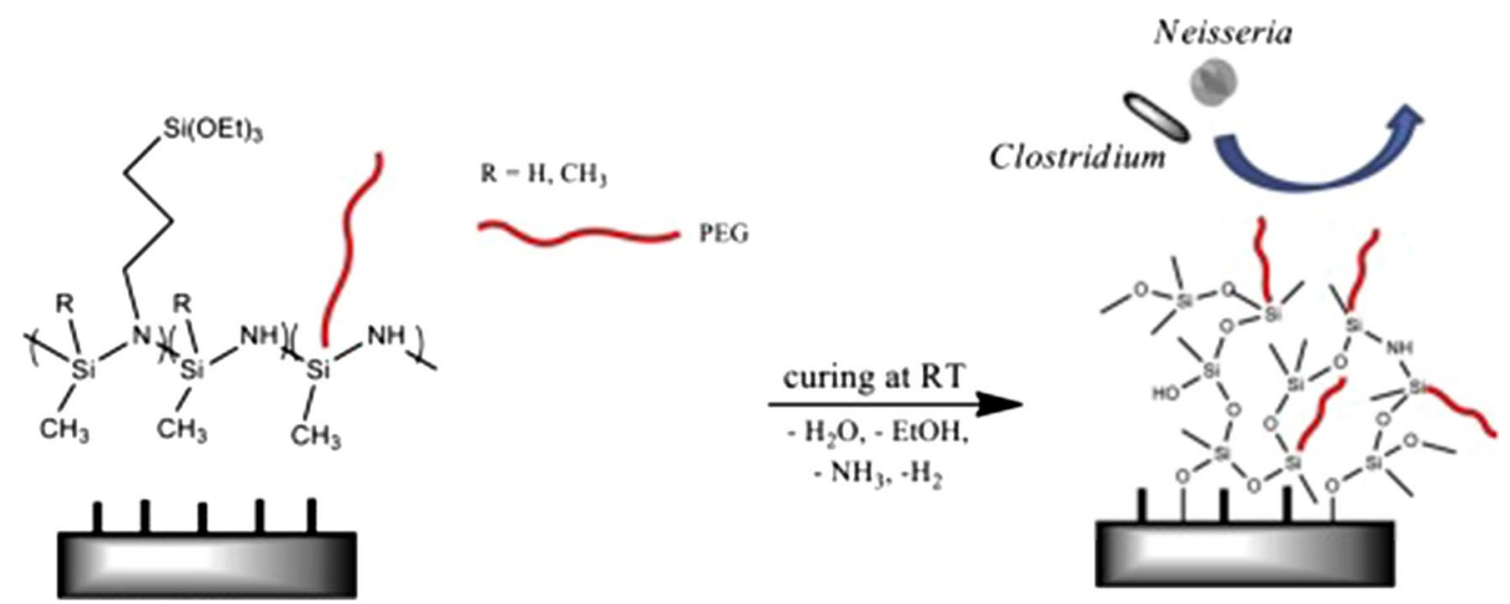

Fig. 4 Silazane networks grafted with PEG branches for the marine biofouling anti-adhesion [39] reproduced with permission from Elsevier 
foulants to be removed or released to maintain long-term anti-fouling properties.

A novel stimuli-responsive material is reported to conquer the degradation and detachment of the grafted antifouling molecules, which can lead to a loss of the antifouling effect. In this study, pH-responsive crosslinked poly(2-vinyl pyridine) (P2VP) films (10-20 nm in thickness) on the surface of Si-wafer were prepared first. Poly(ethylene oxide) (PEO) was next grafted to the surface and inside the P2VP network films. Because P2VP and PEO are miscible, PEO chains can penetrate into the P2VP network. The network of P2VP acts as a reservoir of PEO chains. Because P2VP film has $\mathrm{pH}$-responsive properties (swell and collapse if the $\mathrm{pH}$ value is varied) [50], the prepared polymer network can replenish the lost PEO chains at the network interface (Fig. 5). The rearrangement of polymeric chains due to $\mathrm{pH}$ change provides the basis for the self-healing effect. The antifouling properties of the PEO-grafted films were studied by protein adsorption tests using bovine serum albumin (BSA) and fibrinogen. The results demonstrated that the adsorbed amounts of protein on the PEO-grafted films were negligibly small, and this $\mathrm{pH}$-responsive poly(2vinylpyridine) films with the $3 \mathrm{D}$ grafting of poly(ethylene oxide) demonstrate a fourfold increase in longevity of antifouling behavior [51].

In another study, vertical silicon nanowire $(\mathrm{SiN})$ arrays with uniform lengths and densities have been prepared as a substrate. SiN nanoscale structure has a porous structure and high-surface-to-volume ratio. Because of those advantages in structure, SiN can serve as a good reservoir with a high loading capacity for biocidal agents, and therefore was considered in this study. After the preparation of $\mathrm{SiN}$ nanowire arrays through a reported chemical etching method [52], the nanoscale SiN wires were then modified with a $\mathrm{pH}$-responsive polymer, poly(methacrylic acid) (SiN-PMAA). Because SiNPMAA surfaces exhibit $\mathrm{pH}$-responsive protein adsorption behavior under acidic conditions, Lysozyme, an antibacterial enzyme, was adsorbed on the SiN-PMAA surfaces for use in biocidal applications. It was hypothesized that lysozymes bind to SiN-PMAA surfaces through hydrogen bonds formed between - $\mathrm{COOH}$ groups on PMAA chains and the - $\mathrm{CONH}$ - groups on proteins. When the condition becomes neutral, the $\mathrm{COOH}$ groups on PMAA chains are ionized into $-\mathrm{COO}^{-}$ groups, leading to the breaking of hydrogen bonds and thus reducing the interaction between the proteins and the surfaces. The loaded lysozymes are therefore released to kill the E. coli attached on the surface and suspended in solution near the surface. As the $\mathrm{pH}$ increases from 7 to 10 , the ionization of $-\mathrm{COOH}$ increases resulting in increased negative charge density and farther extension of the polymer chains away from the surface. Those changes in the surface properties lead to the release of the attached dead bacteria from the surface (Fig. 6) [53].

We found that few research papers have reported on the use of stimuli-responsive polymers modified silicon and silicon-based materials for marine applications. Therefore, more research in this area is expected in

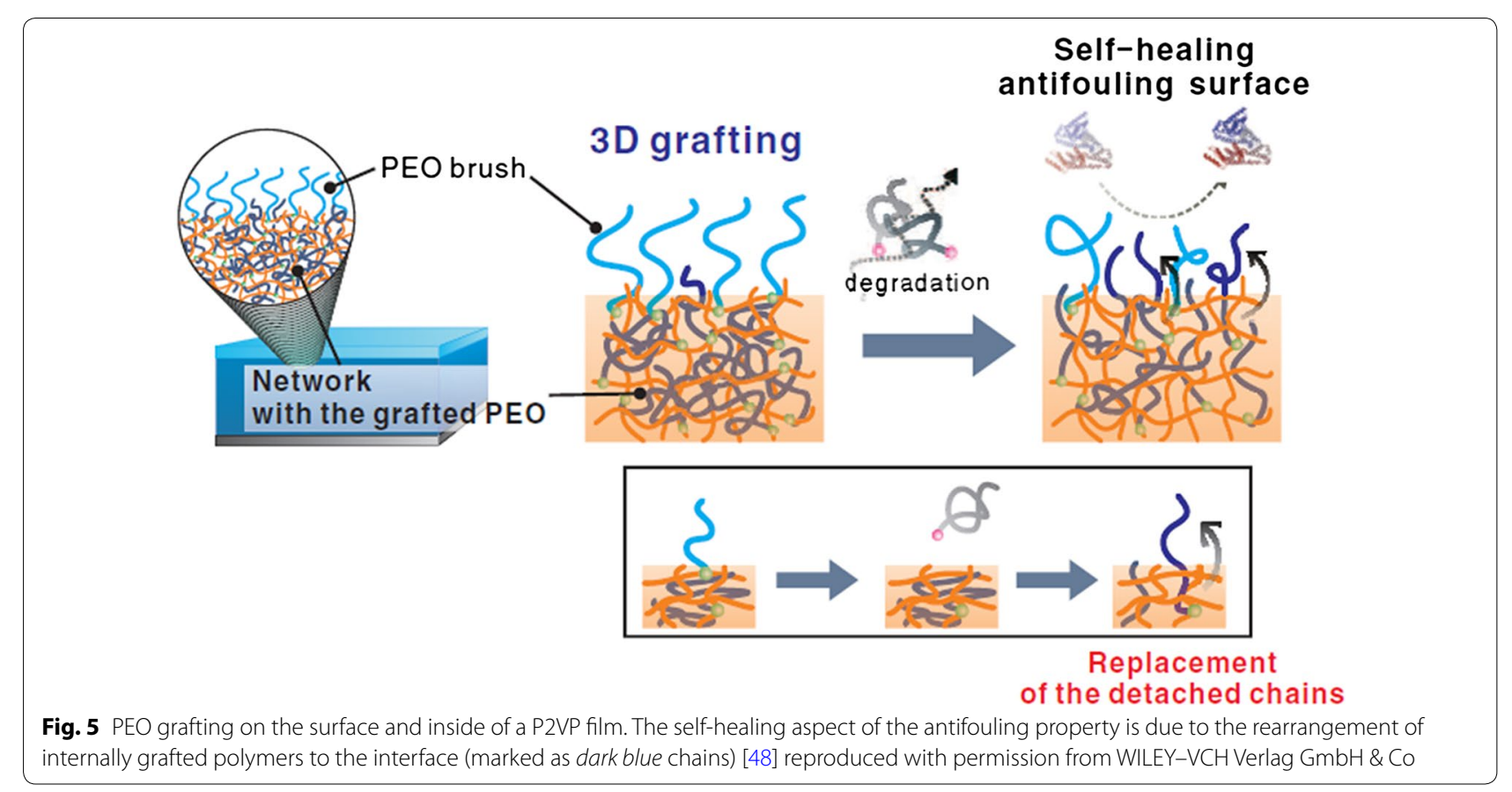




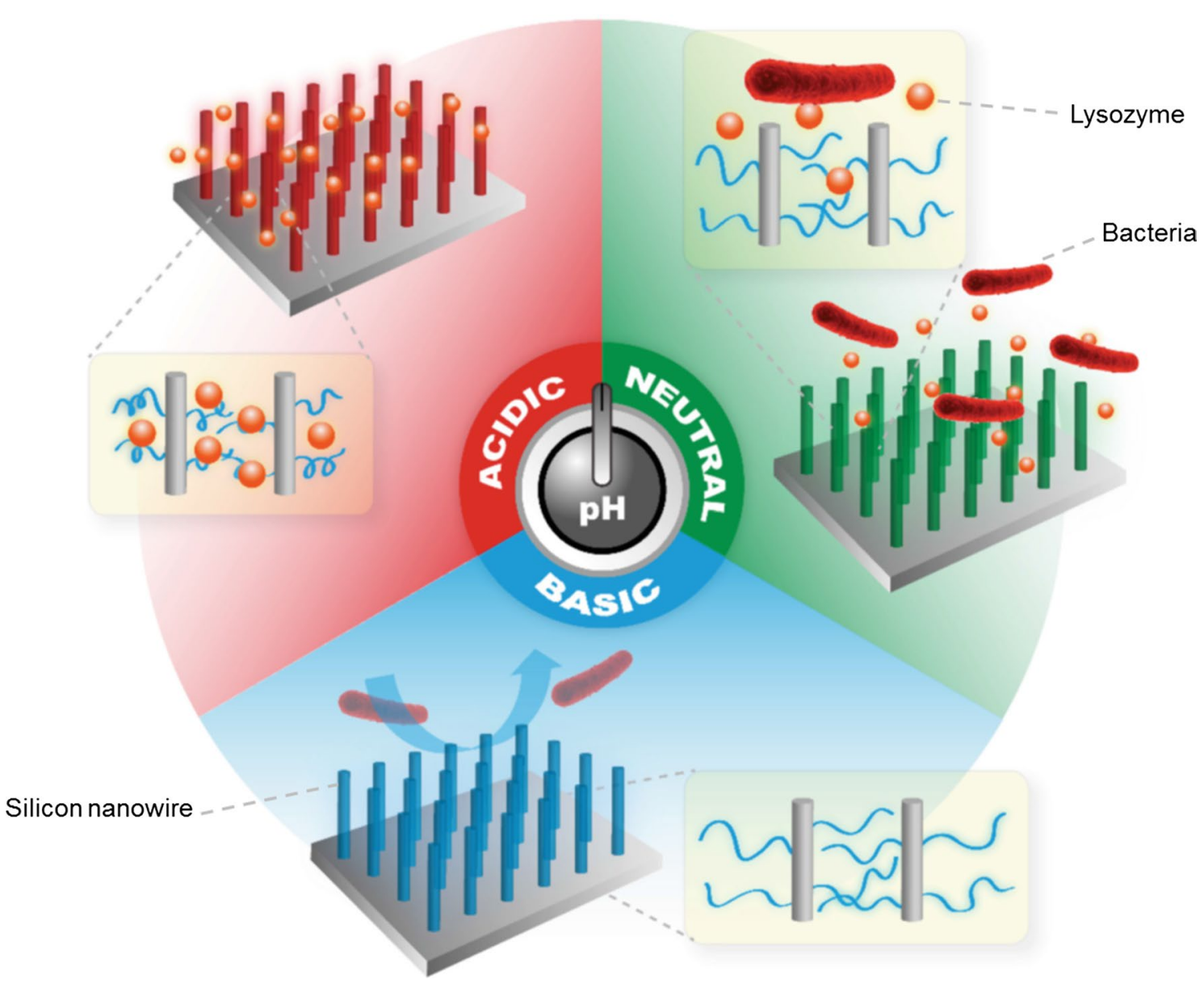

Fig. 6 Schematic illustration of a smart antibacterial surface with pH-responsive capability of loading biocide, killing bacteria, and releasing bacteria [50] reproduced with permission from WILEY-VCH Verlag GmbH \& Co

the near future. An intrinsic difficulty of these stimuliresponsive polymers-coatings is the trade-off between the ability to switch and the mechanical stability of the system. At low crosslink densities, the switching process works well, but the coatings are quite fragile. At high crosslink densities, the coatings are more robust, but switching becomes increasingly difficult [54].

Incorporation of biocidal agents, such as synthetic biocides and enzymatic biocides, on surfaces is an effective approach to kill or degrade attached bacteria, and therefore inhibit their proliferation and formation of biofilms. For example, quaternary ammonium salt (QAS), one type of synthetic biocide, can provide effective protection against bacterial colonization by disrupting the cell membrane through the binding of their ammonium cations to anionic sites in the outer layer tissue of bacteria [21]. It was reported that the QAS-modified substrate can resist the bacterial adhesion with water-repelling hydrophobicity and eradicate the contacted bacteria with biocidal capability [55].
The López lab at Duke University combined the antimicrobial activity of QAS and stimuli-responsive polymers, poly( $N$-isopropylacrylamide) (PNIPAAm), which have been previously shown to controllably release adsorbed organisms [56-60], resulting in effective killing and continued de-attachment of dead bacteria [61]. First, the silicon wafer was cleaned with "Piranha" solution. This process results in the formation of a thin silicon dioxide layer. Then, self-assembled monolayers (SAMs), terminated with atom transfer radical polymerization initiators, was immobilized on the wafer. Interferometric lithography (IL) was then used for the surface patterning. PNIPAAm polymer brushes were then grafted onto the patterned SAMs using surface-initiated activators regenerated by electron transfer-atom transfer radical polymerization. The nanopatterned PNIPAAm surfaces were then incubated in QAS solution to produce hybrid surfaces (QAS was integrated onto the substrate between nanopatterned PNIPAAm brushes, Fig. 7). The prepared surface was tested against $E$. coli K12. The 


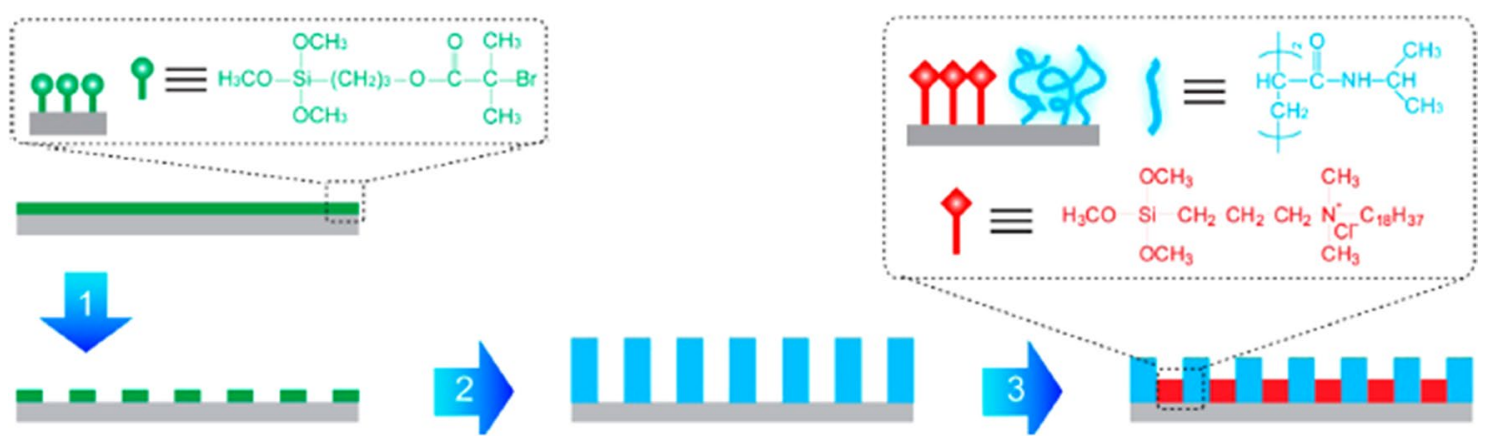

Fig. 7 Schematic depiction of the procedure for the preparation of nanopatterned PNIPAAm surfaces (Steps 1 and 2) and nanopatterned PNIPAAm/QAS Surfaces (Steps 1-3) [60] reproduced with permission from Royal Society of Chemistry

bacteria were killed as they were exposed to QAS moieties. Furthermore, the reduction of the temperature results in the swollen PNIPAAm chain which promotes the release of dead bacteria (Fig. 8). In their later study, lysozyme replaced QAS and served as a biocide. The surface exhibited the same ability to control the attachment, killing and release of bacteria in response to temperature changes. Compare to QAS, lysozyme is environmentally friendly, requires no toxic precursors or cost disposal protocols [62].

\section{Zwitterionic polymers}

Zwitterionic polymers are polymers that have moieties possessing both cationic and anionic groups. These materials are characterized by high dipole moments and highly charged groups but are charge neutral [63]. The strongly bound hydration layer, induced by electrostatically ionic solvation in addition to hydrogen-bonding interactions, is considered to be the reason for the efficient repulsion of fouling materials-the electrostatic interactions between water molecules and dipoles present in the zwitterionic polymer chains make these

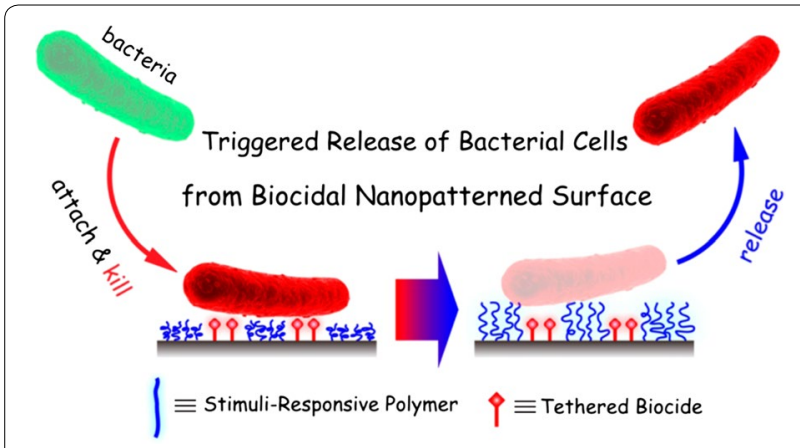

Fig. 8 Upon a reduction of the temperature, swollen PNIPAAm chains promote the release of dead bacteria [60] reproduced with permission from Royal Society of Chemistry polymers better "water-bears" [18, 64, 65]. For example, Huang's group recently modified the silicon-based materials, PDMS, with sulfobetaine silane (SBSi) with covalent silanization. This superhydrophilic zwitterionic interface presents its antibacterial adhesion property to resist nonspecific adsorption of bacteria (S. epidermidis and $P$. aeruginosa), protein (bovine serum albumin, lysozyme, and mucin), and lipids. Moreover, because the cellular liability experiment demonstrated that SBSi had negligible cytotoxicity in vivo application, the applicability of SBSi modification was applied to silicone hydrogel contact lenses by following the same procedure as that for PDMS. The SBSi-modified contact lens was kept under the $P$. aeruginosa solution in a physiological condition. The experimental results demonstrated that the number of adherent bacteria on SBSi-modified contact lens is much less than unmodified one [5].

Most zwitterionic polymers with antifouling functionality are attached to the surface through $\mathrm{Si}-\mathrm{O}-\mathrm{Si}-\mathrm{C}$ [66-68] and Si-O-C linkages [69, 70]. A major disadvantage of these approaches is the limited hydrolytic stability. This may result in the detachment of the zwitterionic polymers and may consequently keep long-term application out of reach. To increase the stability of the attachment, researchers from Netherlands deposited the Si substrates with $\mathrm{Si}_{x} \mathrm{~N}_{4}(\mathrm{x}>3)$ by low-pressure chemical vapor deposition (LPCVD) with a thickness of $150 \mathrm{~nm}$ first. The sulfobetaine methacrylate (SBMA) zwitterionic polymer brushes were then grafted from $\mathrm{Si}_{x} \mathrm{~N}_{4}$ surfaces by controlled surface-initiated atom-transfer radical polymerization (ATRP) through more stable $\mathrm{Si}-\mathrm{C}$ linkage as compared to less stable $\mathrm{Si}-\mathrm{O}-\mathrm{Si}-\mathrm{C}$ and $\mathrm{Si}-\mathrm{O}-\mathrm{C}$ linkages. As a result, the long-term protein-repellent properties of the zwitterionic polymer (polySBMA) remain largely unaffected [71].

Due to the challenges in surface stability and corrosion, zwitterionic polymers have limited use in marine applications [72, 73]. To prevent cleavage of the anchoring 
segment and increase the long-term stability of zwitterionic polymer-based brushes in seawater, Vancso and co-workers investigate diblock copolymer-type brushes composed of bottom hydrophobic segments and a polysulfobetaine top on the substrate of $\mathrm{SiO}_{x}$ (Fig. 9) [74]. The hydrophobic nature of the protecting block limits water penetration into the brush; therefore, the stability of hydrophilic brushes in aqueous media grown from $\mathrm{SiO}_{x}$ substrates are enhanced [75]. The visual appearance of diblock architecture protected zwitterionic brushes did not change after long exposure to seawater (3 months). No sign of microbial growth was observed during the experiment (14 weeks). Atomic Force Microscopy (AFM) topographical measurements were used to measure the surface modified with the diblock copolymer brushes after 4 weeks of immersion, showing almost no differences in surface morphology (Fig. 10).

\section{Fabrication of surfaces with nano- or micro- topographical features}

It is well-known that the surface microstructure influences cell behavior or tissue formation [76], and certain topography features may contribute to a fouling-free surface. In Carman's study, the designated surface microstructures, including ridges and channels, were induced on a silicone elastomer based cross-linked PDMS films by adopting microfabrication techniques. Their results demonstrated that the settlement of Ulva linza zoospores related to the ridge topographies, and is inversely proportional to the width (between 5 and $20 \mu \mathrm{m}$ ) of the channels [77].

Because the special surface topography of skin or shells includes micro- and nanostructure, many marine organisms do not have biofouling [78, 79]; therefore, artificial surfaces with biomimicking natural microtextures have

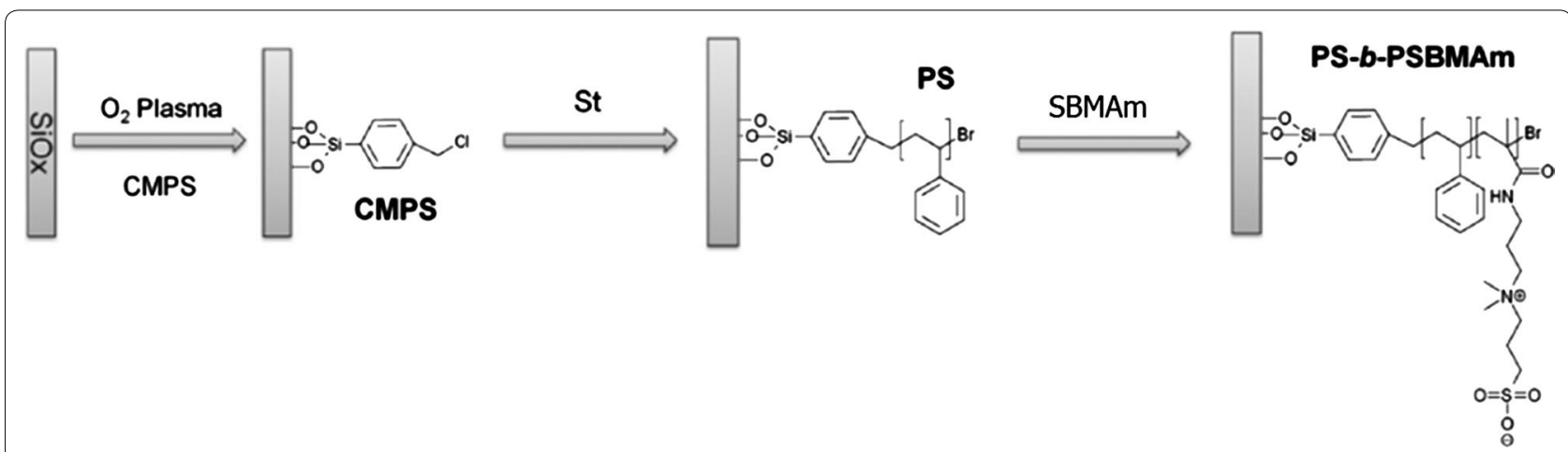

Fig. 9 Synthesis of polymethyl methacrylate (PMMA) and polystyrene (PS) with two hydrophobic polymer blocks between PMMA and PS (PSb-PSBMAm) on $\mathrm{SiO}_{x}$ surface via surface-initiated atom-transfer radical polymerization. CMPS refers to ( $\mathrm{p}$-Chloromethyl)phenyl-trichlorosilane), and SBMAm indicates sulfobetaine methacrylamide [73] reproduced with permission from ACS Publications
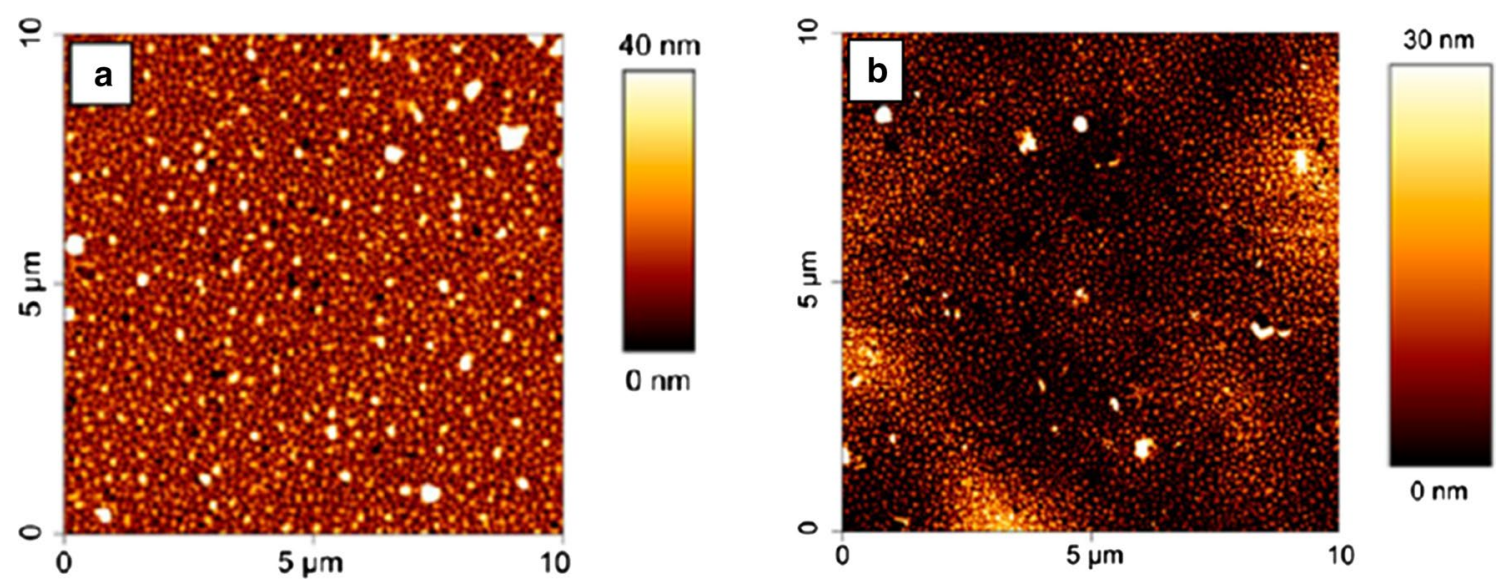

Fig. 10 Tapping-mode AFM images in air of PS-b-PSBMAm block copolymer brushes before (a) and after (b) 4 weeks of immersion in seawater [73] reproduced with permission from ACS Publications 
been fabricated and studied. In a recent work, researchers designed PDMS hierarchical surface microtopographies that mimic the critical features observed on the $M$. hardwickii surface. This micropatterned surface was subject to fouling tests, including laboratory assays against algae adhesion and was also exposed to marine environments during field testing. Their results demonstrated that the settlement of organism on the patterned PDMS was lower than that on the smooth PDMS, indicating that the designed micrographic surface features associate with antifouling. The micropatterned PDMS samples were further modified with zwitterionic polymer brushes, and it was reported that the use of microtopography enhances the antifouling performance of zwitterionic polymer brushes to a greater extent [80].

Another pioneering report of biomimicry focused on the surface of the Trifolium leaf, which has a self-cleaning property. In this work, silicone elastomer was used to fabricate biomimetic surfaces using the Trifolium leaf as a template. The surface of the replica displays a remarkable amount of microspines with a size similar to that of the original Trifolium leaf, and is effective in resisting the settlement of microalgae. The antifouling property of the replica was improved by modification with poly(3-sulfopropyl methacrylate) (PSPMA), a kind of hydrophilic acrylate polymers [81].

In a recent study, Huang et al. [82] fabricated biomimetic surfaces of shark skin onto the surface of PDMS (Fig. 11). BSA and ovalbumin (OVA) were used as a model for protein and glycoprotein to study the surface's anti-protein adhesion properties. The contact angle variation after the surface soaked in protein solution indicates that the fabricated biomimetic surface microstructure can block the adhesion of protein.

\section{Conclusion and perspectives}

We have reviewed strategies for designing effective antifouling approaches for silicon and silicon-based materials, although several of them have associated shortcomings. In addition, by providing a surface topography that is unfavorable for biofoulant attachment, it can also repel the attached biofoulant from the silicon and silicon-based material surface. Although many works claim their antifouling coatings or surface modifications have long-term stability, it is our understanding that an antifouling coating doesn't last forever; as it becomes aged, it becomes less effective. It also has been brought to our attention that once the deposition of foulants has taken place, the surface modification is no longer effective at preventing fouling, which is understandable in light of the fact that the effect of solute/coating interaction is severely reduced once a layer of deposited foulants is formed [83]. In other words, the development of an absolutely nonfouling surface is extremely difficult. The old antifouling coating needs to be removed, and a new antifouling coating needs to be applied once the fouling layer is formed. One of the approaches to remove antifouling paint is by scraping, which is a time consuming process. In addition, one might damage the surface during this coating removal process. We must therefore explore methods by which to restore the permanently fouled surface and maximize the effective use of the modified materials. We expect that these methods can be described in near future and become solutions to reduce the cost associated with fouling for industry, and can prevent long-term bio-fouling for those biomedical devices which are fouled over quickly, such as the colonization of bacteria on catheters, contact lenses, and surgical tools, so that the healthcare costs can be decreased. Besides,

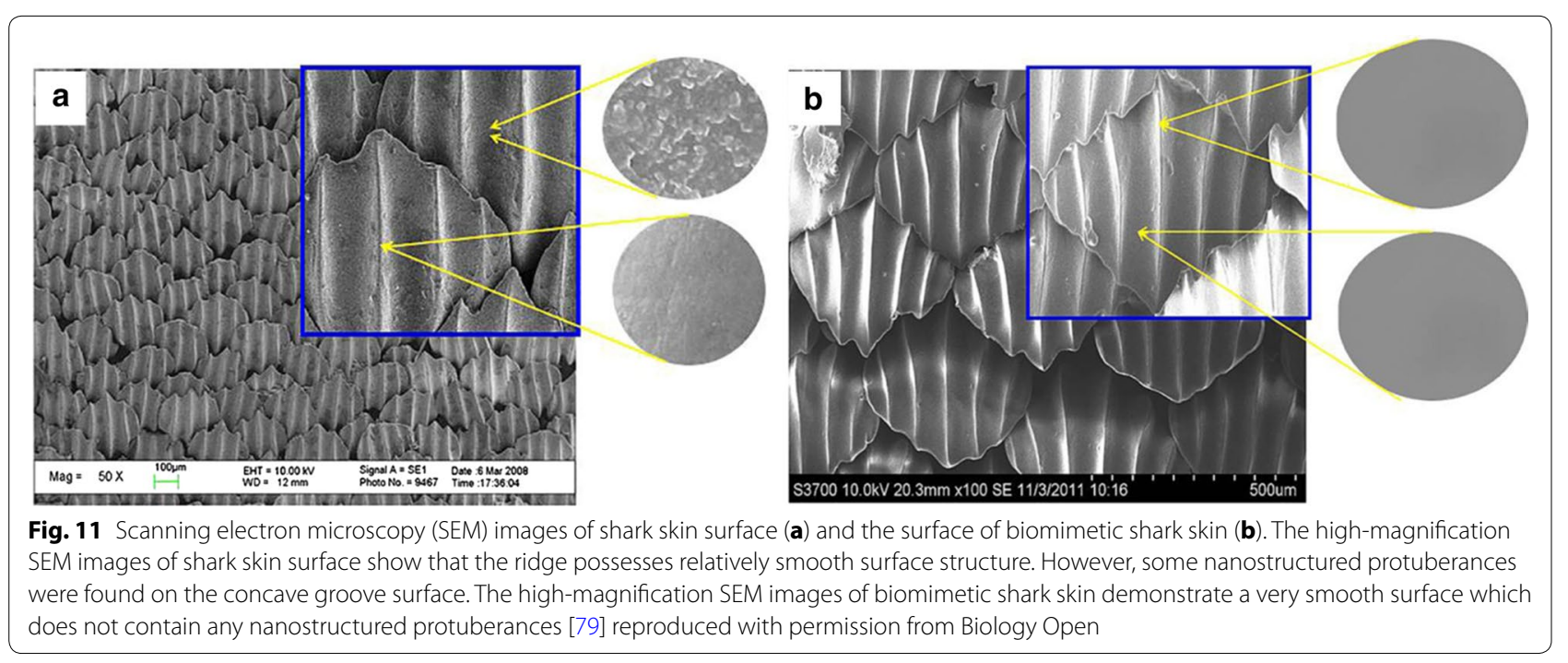


we expect more studies testing the cost-effectiveness, durability, and stability of those designs in real situations, such as the prevention of marine fouling and fouling on medical implants/devices. However, in those cases, the challenge would be developing a coating or an approach of modification that will resist adhesion of all forms of biofouling.

\begin{abstract}
Abbreviations
CAUTI: Catheter-associated urinary tract infection; PDMS: polydimethylsiloxane; PEG: poly (ethylene glycol); EO: ethylene oxide (EO); HAS: human serum albumin (HSA); Lys: lysozyme (Lys); P2VP: poly(2-vinyl pyridine); PEO: poly(ethylene oxide); BSA: bovine serum albumin; SiN: silicon nanowire; PMAA: poly(methacrylic acid); QAS: quaternary ammonium salt; SAMs: self-assembled monolayers; IL: interferometric lithography; PNIPAAm: poly(N-isopropylacrylamide); SAMs: self-assembled monolayers; SBSi: sulfobetaine silane; AFM: Atomic Force Microscopy; LPCVD: low-pressure chemical vapor deposition; PSPMA: poly(3-sulfopropyl methacrylate); OVA: ovalbumin; SEM: Scanning Electron Microscopy.
\end{abstract}

\section{Authors' contributions}

All authors carried out the literature research and the writing of the manuscript. All authors read and approved the manuscript.

\section{Author details}

1 Department of Mathematics, Sciences and Technology, Paine College, 1235 Fifteenth Street, Augusta, GA 30901, USA. ${ }^{2}$ Key Laboratory of Mariculture and Enhancement of Zhejiang Province, Marine Fishery Institute of Zhejiang Province, Zhoushan 316021, China.

\section{Acknowledgements}

The authors gratefully acknowledge the financial support from the National Science Foundation (HRD-1505197). H. H. is grateful for support from the Science and Technology Project of Zhejiang Province (2017F50021) and the Science and Technology Project of Zhoushan City (2016C31055).

\section{Competing interests}

The authors declare that they have no competing interests.

Received: 9 July 2016 Accepted: 14 February 2017

Published online: 20 February 2017

\section{References}

1. Yoda R (1998) Elastomers for biomedical applications. J Biomater Sci Polym Ed 9(6):561-626

2. Voskerician G, Shive MS, Shawgo RS, Hv Recum, Anderson JM, Cima MJ et al (2003) Biocompatibility and biofouling of MEMS drug delivery devices. Biomaterials 24(11):1959-1967

3. La Flamme KE, Popat KC, Leoni L, Markiewicz E, La Tempa TJ, Roman BB et al (2007) Biocompatibility of nanoporous alumina membranes for immunoisolation. Biomaterials 28(16):2638-2645

4. Fine D, Grattoni A, Goodall R, Bansal SS, Chiappini C, Hosali S et al (2013) Silicon micro- and nanofabrication for medicine. Adv Healthcare Mater. 2(5):632-666

5. Yeh S-B, Chen C-S, Chen W-Y, Huang C-J (2014) Modification of silicone elastomer with zwitterionic silane for durable antifouling properties Langmuir 30(38):11386-11393

6. Bermudez VM, Perkins FK (2004) Preparation and properties of clean Si3N4 surfaces. Appl Surf Sci 235(4):406-419

7. Sharma S, Johnson RW, Desai TA (2004) XPS and AFM analysis of antifouling PEG interfaces for microfabricated silicon biosensors. Biosens Bioelectron 20(2):227-239

8. Chu PK, Chen JY, Wang LP, Huang N (2002) Plasma-surface modification of biomaterials. Mater Sci Eng Rep. 36(5-6):143-206
9. Siddiq DM, Darouiche RO (2012) New strategies to prevent catheterassociated urinary tract infections. Nat Rev Urol. 9(6):305-314

10. Topal J, Conklin S, Camp K, Morris V, BalcezakT, Herbert P (2005) Prevention of nosocomial catheter-associated urinary tract infections through computerized feedback to physicians and a nurse-directed protocol. Am J Med Qual 20(3):121-126

11. Maki DG, Tambyah PA (2001) Engineering out the risk for infection with urinary catheters. Emerg Infect Dis 7(2):342-347

12. Hydrex nv (2010) Ship Hull Performance in the Post-TBT Era. The Hydrex Group, Belgium. http://ec.europa.eu/environment/life/project/Projects/ index.cfm?fuseaction=home.showFile\&rep=file\&fil=ECOTEC_Ship_Hull_ Performance.pdf

13. Peres RS, Baldissera AF, Armelin E, Alemán C, Ferreira CA (2014) Marinefriendly antifouling coating based on the use of a fatty acid derivative as a pigment. Mater Res. 17(3):720-727

14. Salwiczek M, Qu Y, Gardiner J, Strugnell RA, Lithgow T, McLean KM et al (2014) Emerging rules for effective antimicrobial coatings. Trends Biotechnol 32(2):82-90

15. Yang C, Ding X, Ono RJ, Lee H, Hsu LY, Tong YW et al (2014) Brush-like polycarbonates containing dopamine, cations, and peg providing a broad-spectrum, antibacterial, and antifouling surface via one-step coating. Adv Mater 26(43):7346-7351

16. Tang J, Han Y, Chen H, Lin Q (2016) Bottom-up fabrication of PEG brush on poly(dimethylsiloxane) for antifouling surface construction. Int J Polym Sci. 2016:5

17. Rambarran T, Gonzaga F, Brook MA, Lasowski F, Sheardown H (2015) Amphiphilic thermoset elastomers from metal-free, click crosslinking of PEG-grafted silicone surfactants. J Polym Sci Polym Chem. 53(9):1082-1093

18. Nguyen AT, Baggerman J, Paulusse JM, van Rijn CJ, Zuilhof H (2011) Stable protein-repellent zwitterionic polymer brushes grafted from silicon nitride. Langmuir 27(6):2587-2594

19. Qin G, Cai C (2009) Oxidative degradation of oligo(ethylene glycol)-terminated monolayers. Chem Commun 34:5112-5114

20. Leckband D, Sheth S, Halperin A (1999) Grafted poly(ethylene oxide) brushes as nonfouling surface coatings. J Biomater Sci Polym Ed 10(10):1125-1147

21. Chan Y-HM, Schweiss R, Werner C, Grunze M (2003) Electrokinetic characterization of oligo-and poly (ethylene glycol)-terminated self-assembled monolayers on gold and glass surfaces. Langmuir 19(18):7380-7385

22. Flavel BS, Jasieniak M, Velleman L, Ciampi S, Luais E, Peterson JR et al (2013) Grafting of poly(ethylene glycol) on click chemistry modified Si(100) surfaces. Langmuir 29(26):8355-8362

23. Jon S, Seong J, Khademhosseini A, Tran NT, Laibinis PE, Langer R (2003) Construction of nonbiofouling surfaces by polymeric self-assembled monolayers. Langmuir 19(24):9989-9993

24. Desai NP, Hossainy SF, Hubbell JA (1992) Surface-immobilized polyethylene oxide for bacterial repellence. Biomaterials 13(7):417-420

25. Roosjen A, de Vries J, van der Mei HC, Norde W, Busscher HJ (2005) Stability and effectiveness against bacterial adhesion of poly(ethylene oxide) coatings in biological fluids. J Biomed Mater Res Appl Biomater. 73B(2):347-354

26. Wagner VE, Koberstein JT, Bryers JD (2004) Protein and bacterial fouling characteristics of peptide and antibody decorated surfaces of PEG-poly (acrylic acid) co-polymers. Biomaterials 25(12):2247-2263

27. Humphries M, Nemcek J, Cantwell JB, Gerrard JJ (1987) The use of graft copolymers to inhibit the adhesion of bacteria to solid surfaces. FEMS Microbiol Ecol 3(5):297-304

28. Krsko P, Kaplan JB, Libera M (2009) Spatially controlled bacterial adhesion using surface-patterned poly (ethylene glycol) hydrogels. Acta Biomater 5(2):589-596

29. Roosjen A, van der Mei HC, Busscher HJ, Norde W (2004) Microbial adhesion to poly (ethylene oxide) brushes: influence of polymer chain length and temperature. Langmuir 20(25):10949-10955

30. Wang Q, Uzunoglu E, Wu Y, Libera M (2012) Self-assembled poly(ethylene glycol)-co-acrylic acid microgels to inhibit bacterial colonization of synthetic surfaces. ACS Appl Mater Interfaces. 4(5):2498-2506

31. Banerjee I, Pangule RC, Kane RS (2011) Antifouling coatings: recent developments in the design of surfaces that prevent fouling by proteins, bacteria, and marine organisms. Adv Mater 23(6):690-718 
32. Tiller J, Sprich C, Hartmann L (2005) Amphiphilic conetworks as regenerative controlled releasing antimicrobial coatings. J Controlled Release 103(2):355-367

33. Voo ZX, Khan M, Xu Q, Narayanan K, Ng BWJ, Bte Ahmad R et al (2016) Antimicrobial coatings against biofilm formation: the unexpected balance between antifouling and bactericidal behavior. Polym Chem. 7(3):656-668

34. Liu SQ, Yang C, Huang Y, Ding X, Li Y, Fan WM et al (2012) Antimicrobial and antifouling hydrogels formed in situ from polycarbonate and poly (ethylene glycol) via Michael addition. Adv Mater 24(48):6484-6489

35. Yebra DM, Kiil S, Dam-Johansen K (2004) Antifouling technology_past, present and future steps towards efficient and environmentally friendly antifouling coatings. Prog Org Coat 50(2):75-104

36. Schultz MP, Swain GW (1999) The effect of biofilms on turbulent boundary layers. J Fluids Eng 121(1):44-51

37. Schultz MP, Swain GW (2000) The influence of biofilms on skin friction drag. Biofouling. 15(1-3):129-139

38. Townsin RL (2003) The ship hull fouling penalty. Biofouling. 19(sup1):9-15

39. Pimentel D, Lach L, Zuniga R, Morrison D (2000) Environmental and economic costs of nonindigenous species in the United States. Bioscience 50(1):53-65

40. Perrin FX, Nguyen TDH, Nguyen DL (2015) Formation, structure and antibacterial activities of silazane networks grafted with poly(ethylene glycol) branches. Prog Org Coat 88:92-105

41. Rosenhahn A, Schilp S, Kreuzer HJ, Grunze M (2010) The role of "inert" surface chemistry in marine biofouling prevention. PCCP. 12(17):4275-4286

42. Islam MR, Gao Y, Li X, Zhang QM, Wei M, Serpe MJ (2014) Stimuli-responsive polymeric materials for human health applications. Chin Sci Bull 59(32):4237-4255

43. Telford AM, James M, Meagher L, Neto C (2010) Thermally cross-linked PNVP films as antifouling coatings for biomedical applications. ACS App Mater Interfaces. 2(8):2399-2408

44. Yang WJ, Pranantyo D, Neoh K-G, Kang E-T, Teo SL-M, Rittschof D (2012) Layer-by-layer click deposition of functional polymer coatings for combating marine biofouling. Biomacromolecules 13(9):2769-2780

45. Dalsin JL, Lin L, Tosatti S, Vörös J, Textor M, Messersmith PB (2005) Protein resistance of titanium oxide surfaces modified by biologically inspired mPEG - DOPA. Langmuir 21(2):640-646

46. Dalsin JL, Hu B-H, Lee BP, Messersmith PB (2003) Mussel adhesive protein mimetic polymers for the preparation of nonfouling surfaces. J Am Chem Soc 125(14):4253-4258

47. Lee KS, In I, Park SY (2014) pH and redox responsive polymer for antifouling surface coating. Appl Surf Sci 313:532-536

48. Meng J, Cao Z, Ni L, Zhang Y, Wang X, Zhang X et al (2014) A nove salt-responsive TFC RO membrane having superior antifouling and easycleaning properties. J Membr Sci 461:123-129

49. Costerton JW, Stewart PS, Greenberg EP (1999) Bacterial biofilms: a common cause of persistent infections. Science 284(5418):1318

50. Elmahdy MM, Drechsler A, Uhlmann P, Stamm M (2016) Swelling and surface interactions of end-grafted poly(2-vinylpyridine) layers in acidic solution: influence of grafting density and salt concentration. Langmuir 32(22):5451-5459

51. Kuroki H, Tokarev I, Nykypanchuk D, Zhulina E, Minko S (2013) Stimuliresponsive materials with self-healing antifouling surface via 3D polymer grafting. Adv Funct Mater 23(36):4593-4600

52. Yu Q, Li X, Zhang Y, Yuan L, Zhao T, Chen H (2011) The synergistic effects of stimuli-responsive polymers with nano- structured surfaces: wettability and protein adsorption. Rsc Adv. 1(2):262-269

53. Wei T, Yu Q, Zhan W, Chen H (2016) A smart antibacterial surface for the on-demand killing and releasing of bacteria. Adv Healthcare Mater. 5(4):449-456

54. Buskens P, Wouters M, Rentrop C, Vroon Z (2013) A brief review of environmentally benign antifouling and foul-release coatings for marine applications. J Coat Technol Res 10(1):29-36

55. Song J, Kong H, Jang J (2011) Bacterial adhesion inhibition of the quaternary ammonium functionalized silica nanoparticles. Colloids Surf B Biointerfaces. 82(2):651-656

56. Yu Q, Chen H, Zhang Y, Yuan L, Zhao T, Li X et al (2010) pH-reversible, high-capacity binding of proteins on a substrate with nanostructure. Langmuir 26(23):17812-17815
57. Yu Q, Zhang Y, Chen H, Wu Z, Huang H, Cheng C (2010) Protein adsorption on poly ( $\mathrm{N}$-isopropylacrylamide)-modified silicon surfaces: effects of grafted layer thickness and protein size. Colloids Surf B Biointerfaces. 76(2):468-474

58. Cooperstein MA, Canavan HE (2009) Biological cell detachment from poly (N-isopropyl acrylamide) and its applications. Langmuir 26(11):7695-7707

59. Liu D, Xie Y, Shao H, Jiang X (2009) Using azobenzene-embedded selfassembled monolayers to photochemically control cell adhesion reversibly. Angew Chem Int Ed 48(24):4406-4408

60. Ista LK, Mendez S, Lopez GP (2010) Attachment and detachment of bacteria on surfaces with tunable and switchable wettability. Biofouling. 26(1):111-118

61. Yu Q, Cho J, Shivapooja P, Ista LK, López GP (2013) Nanopatterned smart polymer surfaces for controlled attachment, killing, and release of bacteria. ACS Appl Mater Interfaces. 5(19):9295-9304

62. Yu Q, Ista LK, López GP (2014) Nanopatterned antimicrobial enzymatic surfaces combining biocidal and fouling release properties. Nanoscale. 6(9):4750-4757

63. Shao Q, Jiang S (2015) Molecular understanding and design of zwitterionic materials. Adv Mater 27(1):15-26

64. Chen S, Zheng J, Li L, Jiang S (2005) Strong resistance of phosphorylcholine self-assembled monolayers to protein adsorption: insights into nonfouling properties of zwitterionic materials. J Am Chem Soc 127(41):14473-14478

65. He Y, Hower J, Chen S, Bernards MT, Chang Y, Jiang S (2008) Molecular simulation studies of protein interactions with zwitterionic phosphorylcholine self-assembled monolayers in the presence of water. Langmuir 24(18):10358-10364

66. Yao Y, Ma Y-Z, Qin M, Ma X-J, Wang C, Feng X-Z (2008) NHS-ester functionalized poly(PEGMA) brushes on silicon surface for covalent protein immobilization. Colloids Surf Biointerfaces. 66(2):233-239

67. Kitano H, Suzuki H, Matsuura K, Ohno K (2010) Molecular recognition at the exterior surface of a zwitterionic telomer brush. Langmuir 26(9):6767-6774

68. Vaterrodt A, Thallinger B, Daumann K, Koch D, Guebitz GM, Ulbricht M (2016) Antifouling and antibacterial multifunctional polyzwitterion/ enzyme coating on silicone catheter material prepared by electrostatic layer-by-layer assembly. Langmuir 32(5):1347-1359

69. Brault ND, Gao C, Xue H, Piliarik M, Homola J, Jiang S et al (2010) Ultra-low fouling and functionalizable zwitterionic coatings grafted onto $\mathrm{SiO}_{2}$ via a biomimetic adhesive group for sensing and detection in complex media. Biosens Bioelectron 25(10):2276-2282

70. Gao C, Li G, Xue H, Yang W, Zhang F, Jiang S (2010) Functionalizable and ultra-low fouling zwitterionic surfaces via adhesive mussel mimetic linkages. Biomaterials 31(7):1486-1492

71. Nguyen AT, Baggerman J, Paulusse JMJ, Zuilhof H, van Rijn CJM (2012) Bioconjugation of protein-repellent zwitterionic polymer brushes grafted from silicon nitride. Langmuir 28(1):604-610

72. Chapman RG, Ostuni E, Takayama S, Holmlin RE, Yan L, Whitesides GM (2000) Surveying for surfaces that resist the adsorption of proteins. J Am Chem Soc 122(34):8303-8304

73. Ostuni E, Chapman RG, Holmlin RE, Takayama S, Whitesides GM (2001) A survey of structure-property relationships of surfaces that resist the adsorption of protein. Langmuir 17(18):5605-5620

74. Quintana R, Gosa M, Jańczewski D, Kutnyanszky E, Vancso GJ (2013) Enhanced stability of low fouling zwitterionic polymer brushes in seawater with diblock architecture. Langmuir 29(34):10859-10867

75. Paripovic D, Klok HA (2011) Improving the stability in aqueous media of polymer brushes grafted from silicon oxide substrates by surfaceinitiated atom transfer radical polymerization. Macromol Chem Phys 212(9):950-958

76. Petronis Š, Berntsson K, Gold J, Gatenholm P (2000) Design and microstructuring of PDMS surfaces for improved marine biofouling resistance. $J$ Biomater Sci Polym Ed 11(10):1051-1072

77. Carman ML, Estes TG, Feinberg AW, Schumacher JF, Wilkerson W, Wilson $\mathrm{LH}$ et al (2006) Engineered antifouling microtopographies-correlating wettability with cell attachment. Biofouling. 22(1):11-21

78. Scardino AJ, Hudleston D, Peng Z, Paul NA, de Nys R (2009) Biomimetic characterisation of key surface parameters for the development of fouling resistant materials. Biofouling. 25(1):83-93 
79. Efimenko K, Finlay J, Callow ME, Callow JA, Genzer J (2009) Development and testing of hierarchically wrinkled coatings for marine antifouling. ACS Appl Mater Interfaces. 1(5):1031-1040

80. Brzozowska AM, Parra-Velandia FJ, Quintana R, Xiaoying Z, Lee SS, ChinSing $L$ et al (2014) Biomimicking micropatterned surfaces and their effect on marine biofouling. Langmuir 30(30):9165-9175

81. Wan F, Pei X, Yu B, Ye Q, Zhou F, Xue Q (2012) Grafting polymer brushes on biomimetic structural surfaces for anti-algae fouling and foul release. ACS Appl Mater Interfaces. 4(9):4557-4565
82. Pu X, Li G, Huang H (2016) Preparation, anti-biofouling and dragreduction properties of a biomimetic shark skin surface. Biol Open. 5(4):389-396

83. Rana D, Matsuura T (2010) Surface modifications for antifouling membranes. Chem Rev 110(4):2448-2471

\section{Submit your manuscript to a SpringerOpen ${ }^{\circ}$ journal and benefit from:}

- Convenient online submission

- Rigorous peer review

- Immediate publication on acceptance

- Open access: articles freely available online

- High visibility within the field

- Retaining the copyright to your article 